\title{
Modeling of Local Magnetic Field Enhancements within Solar Flux Ropes
}

\author{
E. Romashets $\cdot$ M. Vandas $\cdot$ S. Poedts
}

Received: 18 June 2009 / Accepted: 10 December 2009 / Published online: 12 January 2010

(C) Springer Science+Business Media B.V. 2010

\begin{abstract}
To model and study local magnetic-field enhancements in a solar flux rope we consider the magnetic field in its interior as a superposition of two linear (constant $\alpha$ ) forcefree magnetic-field distributions, viz. a global one, which is locally similar to a part of the cylinder, and a local torus-shaped magnetic distribution. The newly derived solution for a toroid with an aspect ratio close to unity is applied. The symmetry axis of the toroid and that of the cylinder may or may not coincide. Both the large and small radii of the toroid are set equal to the cylinder's radius. The total magnetic field distribution yields a flux tube which has a variable diameter with local minima and maxima. In principle, this approach can be used for the interpretation and analysis of solar-limb observations of coronal loops.
\end{abstract}

Keywords Sun: magnetic field $\cdot$ Sun: flares $\cdot$ Sun: coronal mass

\section{Introduction}

As is clear from observations, waves and instabilities are regularly generated in solar magnetic flux ropes or loops. The source of the initial pulse can be impulsive magnetic reconnection at one of the foot points, which causes a local increase of the magnetic flux. Also, an initial instability can be triggered during interactions between two isolated loops (Verwichte, Nakariakov, and Cooper, 2005). In terms of the geometrical size, we consider here the situation when a tube with uniform thickness has a local increase of its diameter at some point,

E. Romashets $(\bowtie)$

Prairie View A\&M University, Prairie View, TX 77446, USA

e-mail: romashets@yahoo.com

M. Vandas

Astronomical Institute, CAS, Prague, Czech Republic

e-mail: vandas@ig.cas.cz

S. Poedts

Center for Plasma Astrophysics, K. U. Leuven, Leuven, Belgium

e-mail: Stefaan.Poedts@ wis.kuleuven.be 
say $z=z_{0}$, here $z$ is the distance along the axis (central line) of the flux rope. Then, as the thicker part moves along the tube, the foot points serve as mirrors, reflecting the motion as solid surfaces do. The period of these swings is of the order of $T=2 L / V$, with $L$ the length of the loop and $V$ the speed of propagation. In the linear approximation, this period does not depend on the thickness of the tube nor on the thickness of the disturbed part. Such perturbations, known as sausage modes, can be described by studying axisymmetric total pressure perturbations of an equilibrium. In the present article, however, we consider a static solar magnetic flux rope with a local irregularity in its shape and we develop a mathematical tool to describe and study such local enhancements.

The idea to treat local spatial disturbances in solar magnetic flux ropes, which have a force-free structure in their interior, as a linear combination of a few linear force-free solutions, was discussed many years ago (Chandrasekhar and Kendall, 1957). More recently, Sakai and de Jager (1997) modeled the process of interaction between two flux ropes by means of a three-dimensional time-dependent magnetohydrodynamic solver and the initial magnetic field was a superposition of two cylindrical force-free fields.

A natural way to describe such a magnetic structure is to impose a compact toroid onto a preexisting cylindrical configuration. However, the problem with this approach is that available toroidal solutions (Miller and Turner, 1981; Ishibashi and Marubashi, 2004) cannot be used if the aspect ratio $\left(R_{0} / r_{0}\right)$ is close to unity. As a matter of fact, in that case the formula of Miller and Turner (1981) does not satisfy both constraints $\nabla \cdot \mathbf{B}=0$ (solenoidality) and $\mathbf{B} \times(\nabla \times \mathbf{B})=0$ (force-free condition). Also the Ishibashi and Marubashi (2004) formulae do not satisfy the solenoidality condition in that limit. Moreover, if one selects only the toroidal-type harmonics from Chandrasekhar and Kendall (1957), they have singularities at the center of symmetry of the toroid and thus these cannot be used either. An attempt to improve the Miller - Turner solution was made by Romashets and Vandas (2003). This solution becomes divergence-free, but it is difficult to make it force-free at small aspect ratios. Recently, Romashets and Vandas (2009) found a toroidal force-free distribution that is applicable for any value of the aspect ratio and is finite everywhere; the solenoidal condition is fulfilled because it is a linear force-free field. This solution is used here to model disturbances in cylindrical flux ropes.

\section{Linear Force-Free Magnetic Field in Toroidal Symmetry}

Romashets and Vandas (2009) described a procedure on how to construct a toroidal linear force-free distribution. Here we only mention their basic concept and results that are important for the derivations we describe. Consider a toroid with a small radius $\left(r_{0}\right)$ and a large radius $\left(R_{0}\right)$ described in Cartesian coordinates $(x, y, z)$ centered in the symmetry point $(\mathrm{O})$ of the toroid and with the $z$-axis along its symmetry axis (see Figure 1). Let us imagine a cylinder with radius $r_{0}$, whose axis is tangential to the circular axis of the toroid at point $T$ (Figure $1 ;|O T|=R_{0}$ ). The position of $T$ is given by the angle $\psi$. The magnetic field inside this cylinder is a linear axially symmetric force-free field: the so-called Lundquist (1950) solution. In local cylindrical coordinates $\left(r^{\prime}, \varphi^{\prime}, z^{\prime}\right)$ with the origin $T$ and the $z^{\prime}$-axis oriented along the cylinder's axis (see Figure 1), the components read

$$
\begin{aligned}
& B_{r^{\prime}}^{\prime}=0, \\
& B_{\varphi^{\prime}}^{\prime}=B_{0} \mathrm{~J}_{1}\left(\alpha r^{\prime}\right), \\
& B_{z^{\prime}}^{\prime}=B_{0} \mathrm{~J}_{0}\left(\alpha r^{\prime}\right),
\end{aligned}
$$


Figure 1 Illustration of how a toroidal field is constructed.

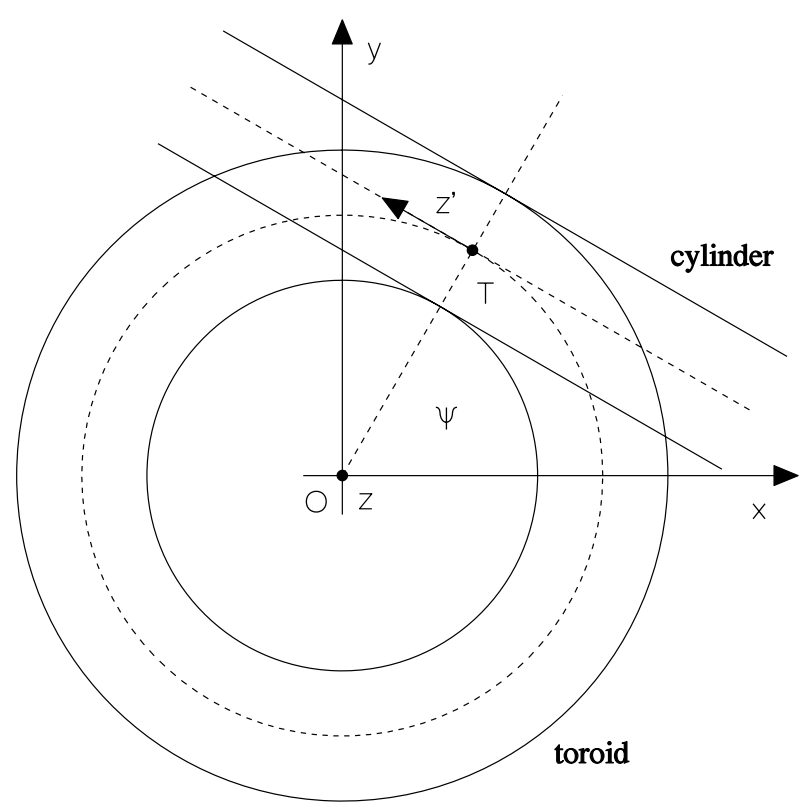

where $\mathrm{J}_{0}$ and $\mathrm{J}_{1}$ are Bessel functions. The solution satisfies the force-free condition $\nabla \times \mathbf{B}=$ $\alpha \mathbf{B}$ with $\alpha=$ const. The sign of $\alpha$ determines chirality (handedness) and the absolute value of $\alpha$ is related to the cylinder's radius by $r_{0}=2.41 /|\alpha|$, where 2.41 is approximately the first root of $\mathrm{J}_{0}$. The absolute value of $B_{0}$ is a maximum field magnitude (located at the cylinder's axis) and the sign of $B_{0}$ determines the field direction. In the global system, the magneticfield components can be expressed as functions of $x, y, z$, and $\psi$ (see Figure 1). The toroidal solution is constructed as a sum of many of these cylindrical solutions in cylinders whose axes are tangential to the toroid's axis. More specifically, each cylindrical contribution is considered as infinitesimal and proportional to $\mathrm{d} \psi$ and the resulting field is obtained as their integration over $\psi$ (see Romashets and Vandas, 2009, for details):

$$
\begin{aligned}
& B_{x}=B_{0} \int_{0}^{2 \pi}\left[\mathrm{J}_{1}(\alpha \rho) \frac{z \cos \psi}{\rho}-\mathrm{J}_{0}(\alpha \rho) \sin \psi\right] \mathrm{d} \psi, \\
& B_{y}=B_{0} \int_{0}^{2 \pi}\left[\mathrm{J}_{1}(\alpha \rho) \frac{z \sin \psi}{\rho}+\mathrm{J}_{0}(\alpha \rho) \cos \psi\right] \mathrm{d} \psi, \\
& B_{z}=-B_{0} \int_{0}^{2 \pi} \mathrm{J}_{1}(\alpha \rho) \frac{x \cos \psi+y \sin \psi-R_{0}}{\rho} \mathrm{d} \psi,
\end{aligned}
$$

where $\rho=\sqrt{\left(x \cos \psi+y \sin \psi-R_{0}\right)^{2}+z^{2}}$. The formulae in Equations (4) through (6) are evaluated numerically.

Figures 2 and 3 show the magnetic structure obtained from these formulae. The magneticfield lines lie in surfaces given by $r B_{\varphi}=$ const., where $B_{\varphi}$ is a magnetic-field component of the field in Equations (4) through (6) in cylindrical coordinates, i.e., $r=\sqrt{x^{2}+y^{2}}$ and $\varphi=$ $\arctan (y / x)$. This can be demonstrated by the following calculation. Due to the symmetry of Equations (4) through (6) with respect to rotation around the $z$-axis, all components do not depend on $\varphi$ (the field is axially symmetric). In addition, the magnetic field satisfies the 
Figure 2 Contours of the magnetic-field magnitude on the toroid's cross section for the following choice of parameter values: $R_{0}=9, r_{0}=3, B_{0}=20$, and $\operatorname{sign}(\alpha)=1$. The black lines correspond to projections of magnetic-field lines (around the rotational axis $z$ the lines are constructed as contours of $r B_{\varphi}=$ const. and the magnetic-field lines are helically twisted on these contours).

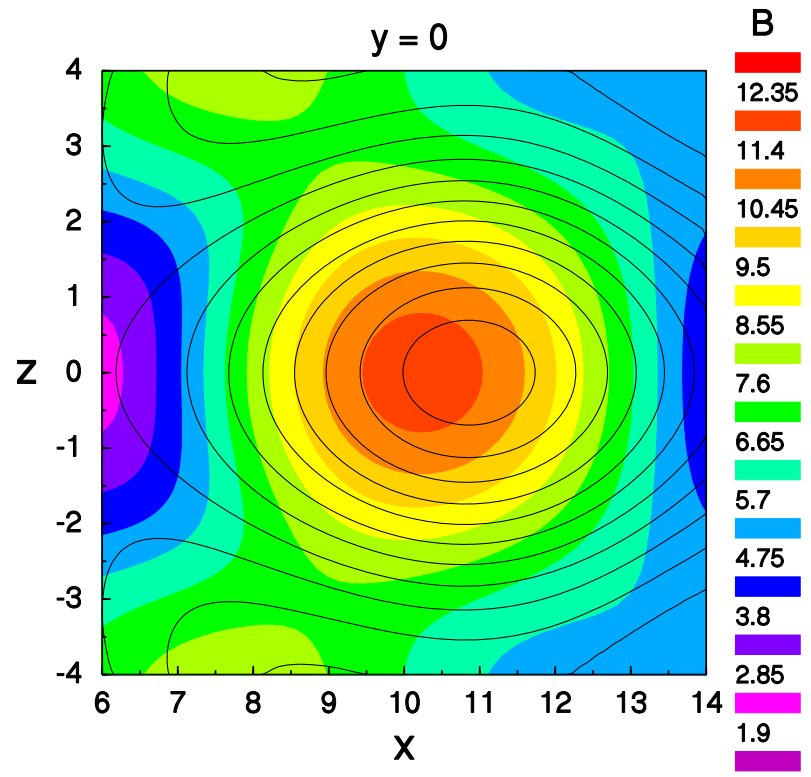

0.95

force-free condition $\nabla \times \mathbf{B}=\alpha \mathbf{B}$. Therefore, we can write

$$
\begin{aligned}
B_{r} & =-\frac{1}{\alpha r} \frac{\partial\left(r B_{\varphi}\right)}{\partial z}, \\
B_{\varphi} & =\frac{1}{\alpha}\left(\frac{\partial B_{r}}{\partial z}-\frac{\partial B_{z}}{\partial r}\right), \\
B_{z} & =\frac{1}{\alpha r} \frac{\partial\left(r B_{\varphi}\right)}{\partial r} .
\end{aligned}
$$

Since there is no dependence on $\varphi$, the following expression should be zero

$$
\begin{aligned}
\mathbf{B} \cdot \nabla\left(r B_{\varphi}\right) & =B_{r} \frac{\partial\left(r B_{\varphi}\right)}{\partial r}+B_{z} \frac{\partial\left(r B_{\varphi}\right)}{\partial z} \\
& =-\frac{1}{\alpha r} \frac{\partial\left(r B_{\varphi}\right)}{\partial z} \frac{\partial\left(r B_{\varphi}\right)}{\partial r}+\frac{1}{\alpha r} \frac{\partial\left(r B_{\varphi}\right)}{\partial r} \frac{\partial\left(r B_{\varphi}\right)}{\partial z}=0 .
\end{aligned}
$$

Figure 2 displays filled contours of the magnetic-field magnitude on the toroid's cross section, i.e., a cut in the $(x, z)$ plane. Actually, in this figure only part of the cross section is shown viz. the part close to the central magnetic axis of the toroid (the axis crosses the point $x=R_{0}=9, z=0$ ).

In Figure 3, filled contours of the same magnetic-field magnitude are displayed on the entire cross section of the toroid (i.e., a cut in the $(x, z)$ plane), including the center of the toroid and the surrounding parts. From this picture it is clear that the magnetic field is symmetric around the $z$-axis and regular everywhere. 
Figure 3 The magnetic-field magnitude on the toroid (and on its neighborhood) on a cut in the $(x, z)$ plane for the same choice of parameters as in Figure 2. We horizontal axis $-20 \leq x \leq 20$, vertical axis $-20 \leq z \leq 20$. show here a larger domain:

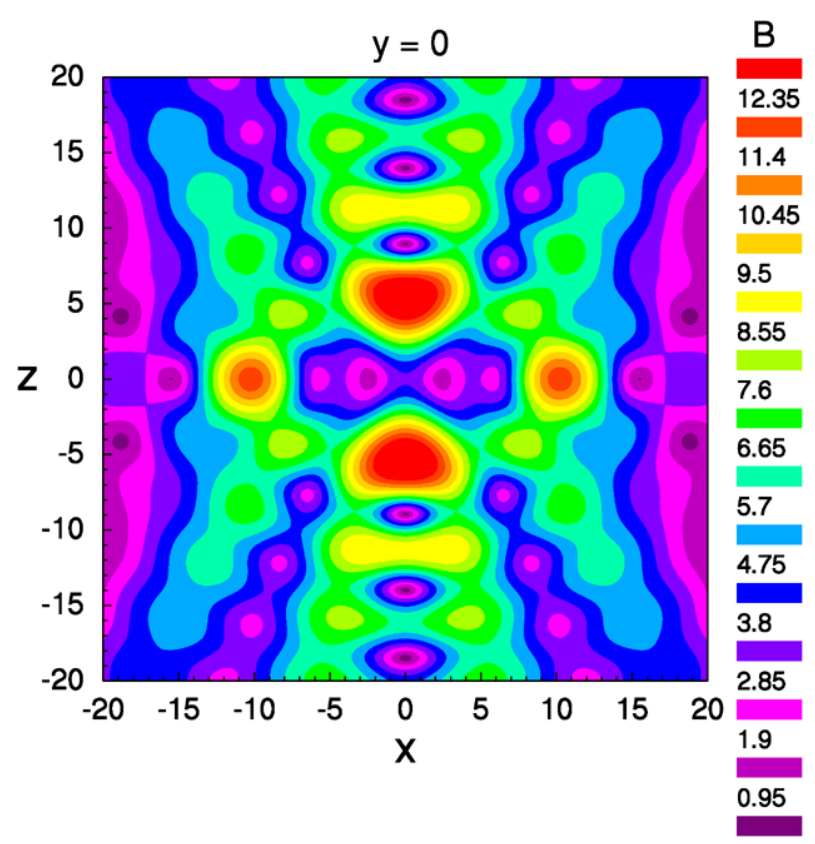

Figure 4 Illustration of how a disturbed field is constructed.

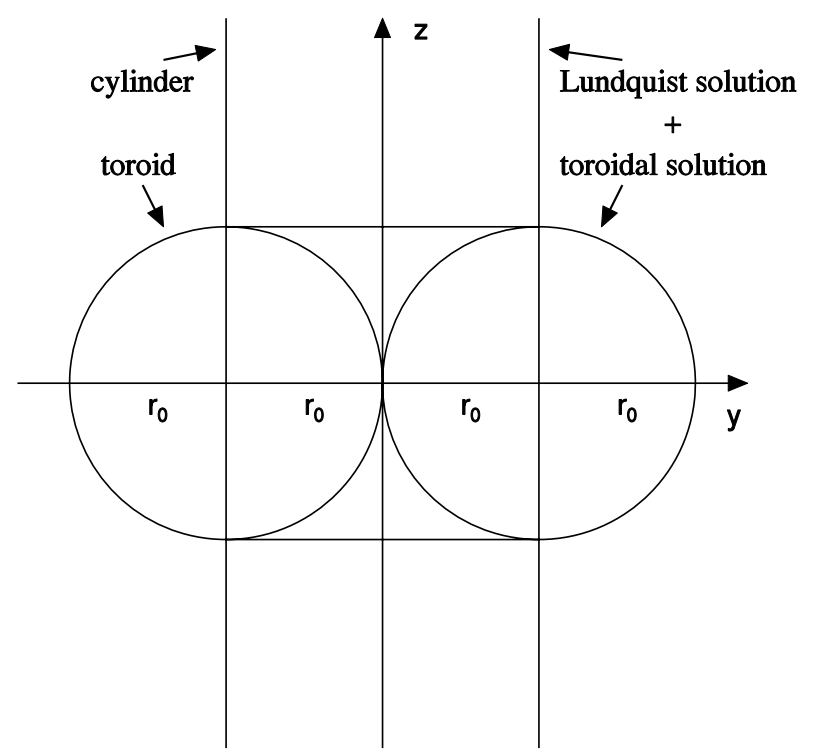

\section{Magnetic Field within Disturbed Flux Ropes}

We now consider a cylindrical force-free tube with a constant- $\alpha$ magnetic field. The cylinder's axis is oriented along the $z$-axis of our global system (Figure 4). Its magnetic field is described by the formulae in Equations (1) through (3) with $B_{00}$ instead of $B_{0}$ and coordinates $r, \varphi$, and $z$, i.e., without primes. The considered tube has a radius $r_{0}$. 
Figure 5 Magnetic-field magnitude distribution (i.e., contour lines of $|B|)$ on a longitudinal cross section of a cylindrical tube, disturbed by an insertion of a compact torus at the center of the coordinate system. The geometric situation corresponds to Figure 4 and the parameters' values for this particular case are $R_{0}=3$, $r_{0}=3, B_{00}=20, B_{0}=2$, and $\operatorname{sign}(\alpha)=1$. The black lines correspond to cross sections of magnetic-flux surfaces (around the rotational axis $z$; the lines are constructed as contours of $r B_{\varphi}=$ const.).

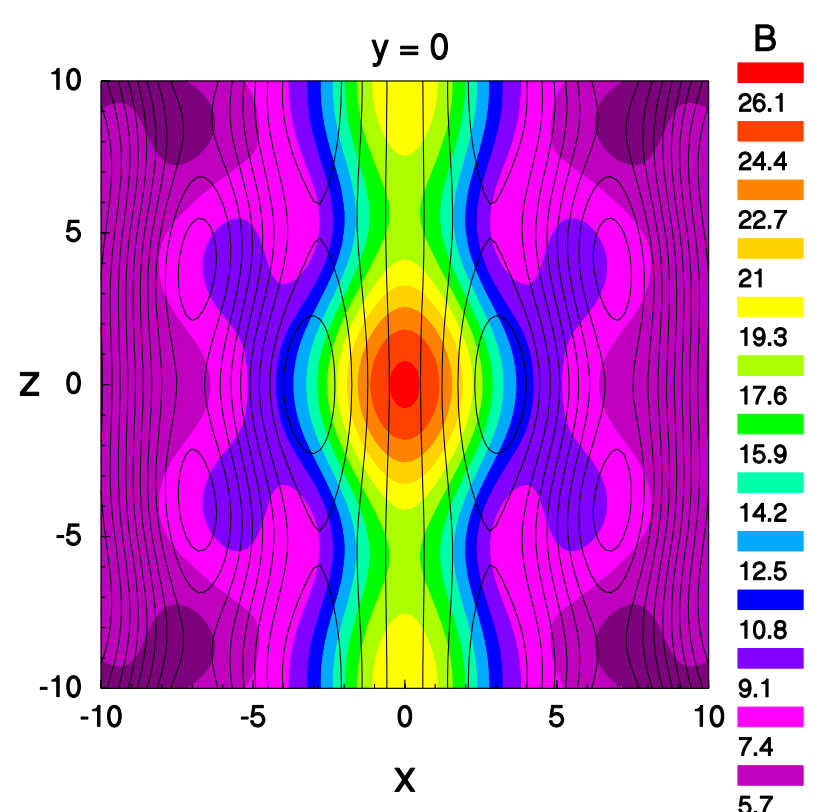

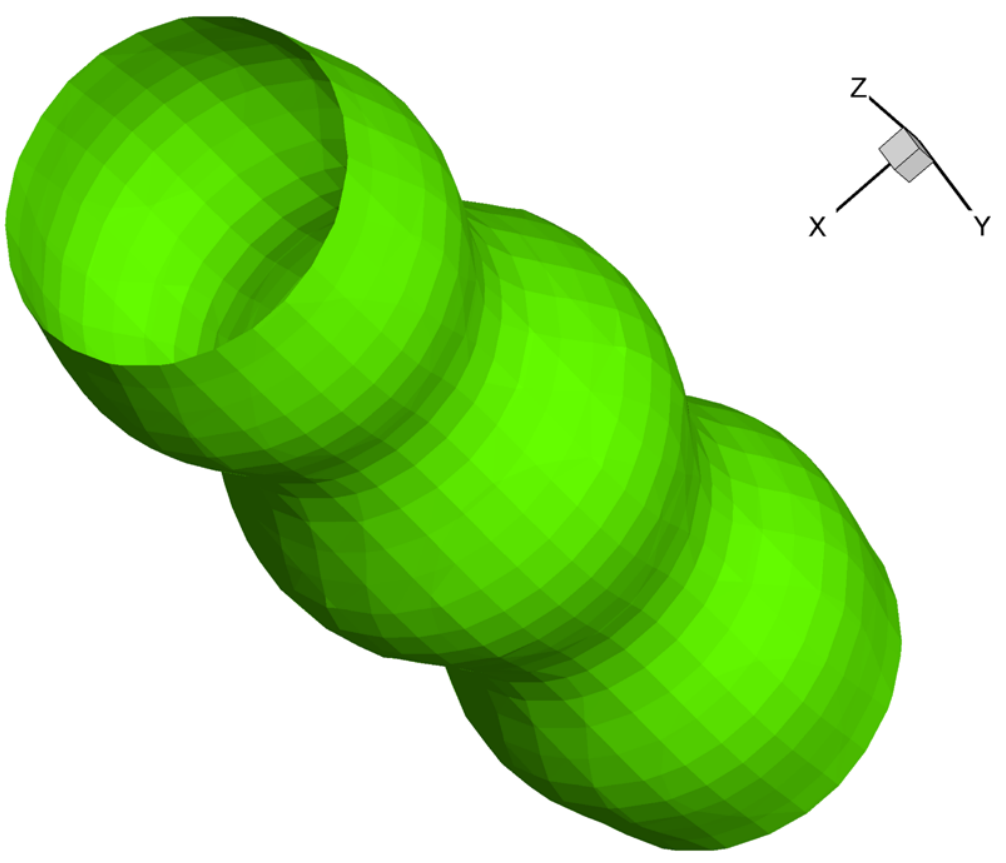

Figure 6 Outer boundary of the tube given by $r B_{\varphi}=$ const. for the case illustrated in Figures 4 and 5 . 
Figure 7 Magnetic-field lines inside the flux tube for the case illustrated in Figures 4 through 6.



To model magnetic disturbances inside this cylindrical flux rope, we superpose on its field a magnetic field determined by a compact toroid (i.e., with $R_{0}=r_{0}$ ), using the solution given by Equations (4) through (6) from the previous section with the same value of $\alpha$. Therefore, the resulting magnetic field is also force-free with (the same) constant $\alpha$. Figure 4 illustrates the situation where the rotational symmetry axis of the toroid coincides with the cylinder axis. However, this is not necessary in general. In the following figures we illustrate the resulting modification of the flux tube obtained by the insertion (superposition) of the compact-toroid solution at some specified point in the cylindrical flux rope.

In Figure 5, the magnetic-field magnitude is shown on a longitudinal cross section of a cylindrical tube of radius 3 , as an example of an application of the newly derived equations and solutions. The magnetic-field strength in the cylinder has $B_{00}=20$. The field is modified by a superposed disturbance in the form of a small toroid at the center of the coordinate system $(0,0,0)$ and with small and large radii both equal to 3 ; the field strength of $B_{0}=2$ is small because this additional field is regarded as a perturbation. The symmetry axis of the superposed toroid coincides with the symmetry axis of the flux tube. The geometric situation thus corresponds to the one illustrated in Figure 4. The magnetic-field configuration is axially symmetric and the magnetic-field lines lie in surfaces given by $r B_{\varphi}=$ const. (see the previous section). Also shown in the figure are cross sections of magnetic-flux surfaces in the plane of the longitudinal cross section. 


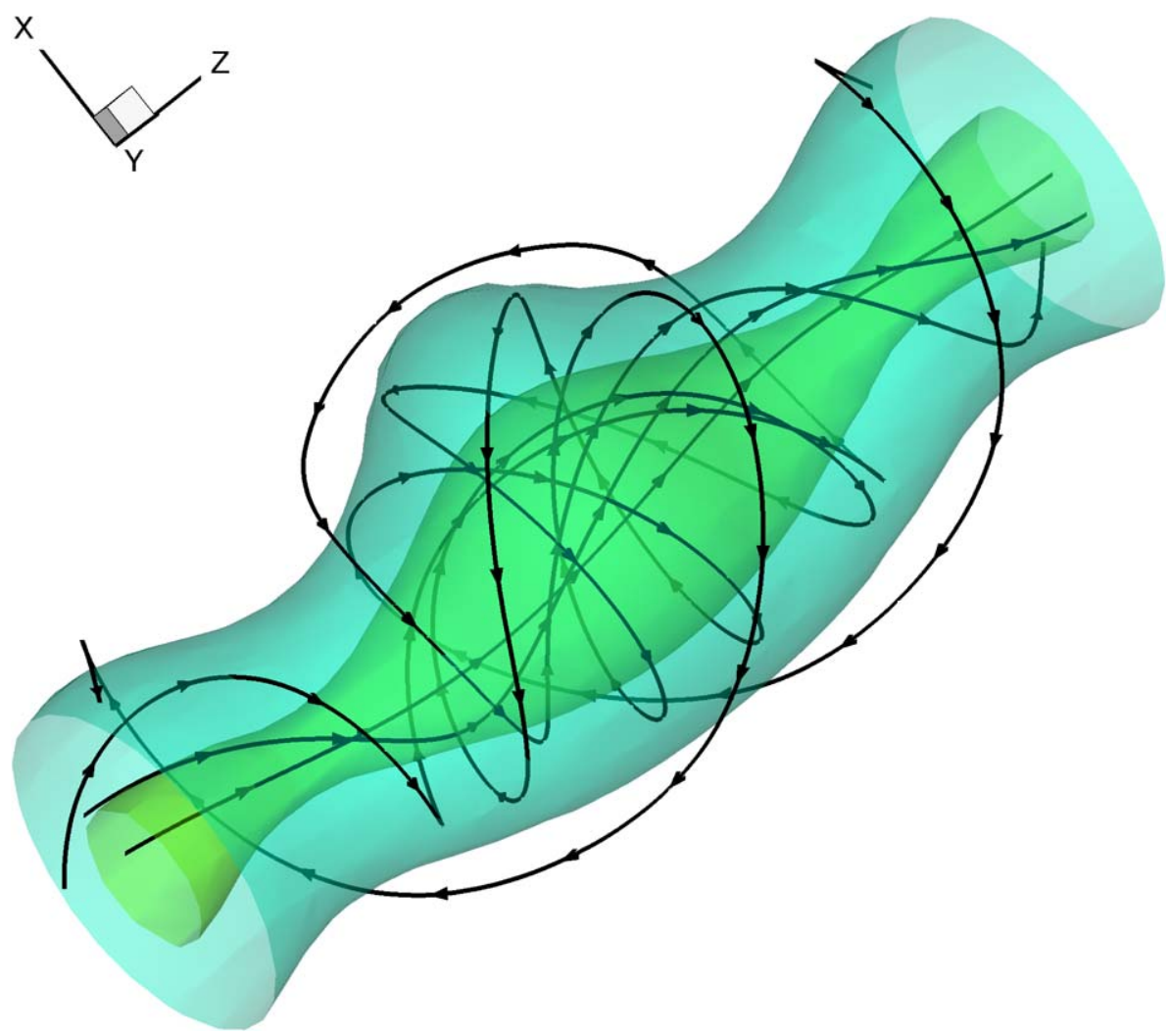

Figure 8 Magnetic-field lines inside a disturbed tube. Here, the axis of symmetry of the toroid is oriented at an angle $\pi / 6$ to the axis of the cylinder. Two plotted surfaces have constant magnetic-field magnitude, with $B=11.5$ and $B=18$, respectively.

In Figure 6, the "boundary" of the disturbed cylindrical tube is shown for the case illustrated in Figures 4 and 5. This "boundary" is defined by $r B_{\varphi}=$ const. The superposed torus-shaped perturbation is clearly visible as the thickening in the middle of the tube. Figure 7 displays a three-dimensional view on the magnetic-field lines inside the disturbed tube, again for the same case as in Figures 4 through 6.

A result for a more complex case is illustrated in Figure 8. This is a full three-dimensional perturbation of a cylindrical flux tube. In this case, the symmetry axis of the inner toroid is inclined with respect to the axis of the cylinder. The inclination angle between the axis of symmetry of the toroid and the axis of the cylinder is $\pi / 6$. Several magnetic-field lines inside the disturbed tube are shown. Because the magnetic-field configuration is not axisymmetric in this case, constant values of $r B_{\varphi}$ cannot be used anymore for tracing the magnetic-flux surfaces. Instead, two isosurfaces of the magnetic-field magnitude are shown in Figure 8.

\section{Conclusions and Discussion}

New mathematical formulae describing a force-free magnetic field with a constant $\alpha$ inside a compact toroid were applied for modeling static magnetic disturbances inside solar 


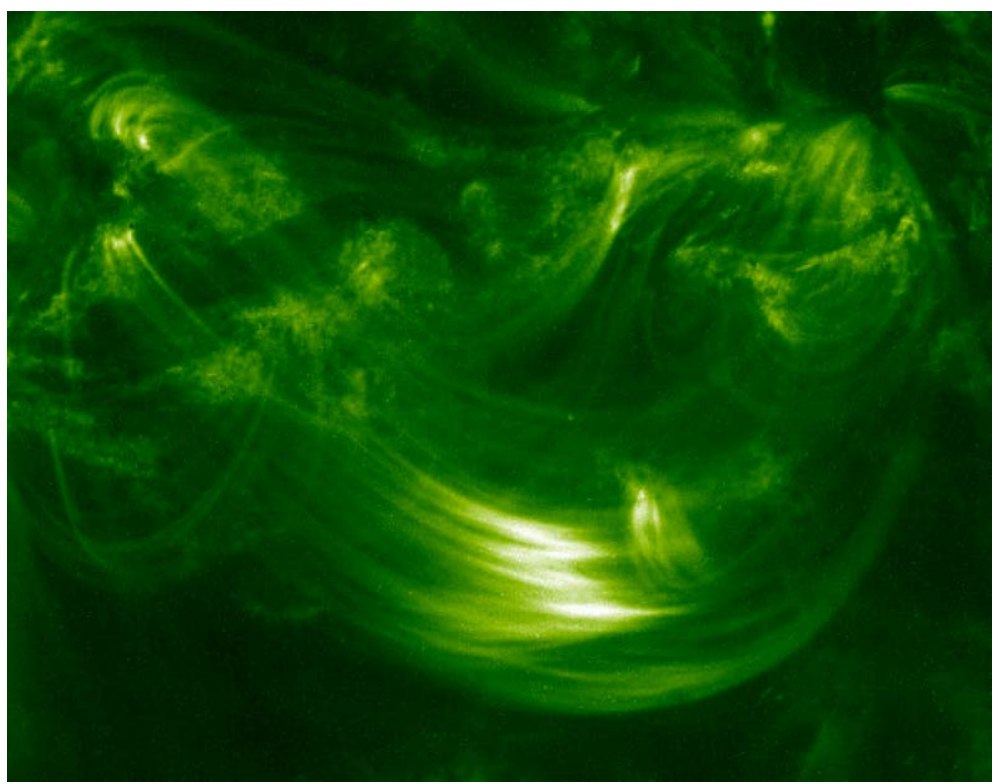

Figure 9 An image of a coronal loop with a bright loop top emission, taken by the TRACE.

magnetic flux ropes. The new compact-toroid solution was superposed onto the well-known force-free cylindrical solution. This description or technique can lead to a better insight and hopefully an explanation of the mechanism and the dynamics of the different irregularities that are frequently observed inside such magnetic flux ropes in the solar corona. Within the proposed approach, such local enhancements are considered as a superposition of a compact toroid with the cylindrical tube. This result should be considered as a mathematical "tool" that can be applied (or used) in the near future to interpret observations of local enhancements in solar magnetic flux tubes. One such possible application where this new tool will be very useful is the study of the drop-off of the magnetic field with height in solar coronal loops and the role of this drop-off for the onset of the torus instability as a triggering mechanism for so-called coronal mass ejections in the flux cancelation model. Another application of the obtained solution can be the interpretation of coronal loop "irregularities" such as the one shown in Figure 9 (Source: http://soi.stanford.edu/results/SolPhys200/Schrijver/ images/T171_001118_155346.gif), which displays a bright loop top emission observed with Transition Region and Coronal Explorer (TRACE) (171 ^; 1 million degrees). Such local emissions are often the consequence of rapid loop cooling taking place within 15 minutes or so. It will be interesting to reveal the magnetic structure that enables such local cooling events. Our new model can be helpful for this, even though it is only (quasi) stationary.

Another clear application of the new "tool" will be to reveal the internal structure of irregularly shaped magnetic clouds related to interplanetary coronal mass ejections. The currently used "standard" model is characterized by a circular cross section, but this model frequently yields problems when applied to the analysis or interpretation of spacecraft data of the passage of such interplanetary magnetic clouds. It is to be expected that the application of this new tool can solve these problems and yield new and better insights into the internal magnetic (and plasma) structure of the clouds, and hence, the resulting signals measured by the spacecraft instruments. 
The solution presented in the present article yields a static picture (description) of the magnetic field. It is not clear at the moment if an equilibrium plasma distribution (including pressure, temperature, and velocity distributions) can be constructed for this magnetic-field configuration, such as in similar previous works (see, e.g., Romashets and Poedts, 2007; Dalakashvili et al., 2009). To obtain a time-dependent description of such disturbances, which can involve motion, expansion, or even the swing of a small toroid on the tube, one should solve the system of time-dependent magnetohydrodynamics (MHD) equations.

Acknowledgements The authors wish to express sincere thanks to many colleagues for useful discussions, as well as very useful suggestions of the referees. The work was supported by BELSPO, RFBR grant 0605-64500 and EU/INTAS grant 03-51-6206. These results were obtained in the framework of the projects GOA/2009-009 (K. U. Leuven), G.0304.07 (FWO-Vlaanderen), and C 90347 (ESA Prodex 9). This work was supported by the program of the Czech - U.S. collaboration in science and technology (ME09032). M.V. acknowledges the support by the AV ČR project 1QS300120506 and by the GA ČR grant 205/09/0170. Image courtesy of the TRACE (NASA/SMEX) team (Figure 9).

\section{References}

Chandrasekhar, S., Kendall, P.C.: 1957, On force-free magnetic fields. Astrophys. J. 126, $457-460$.

Dalakashvili, G., Poedts, S., Fichtner, H., Romashets, E.: 2009, Characteristics of magnetized plasma flow around stationary and expanding magnetic clouds. Astron. Astrophys. 507, 611-616.

Ishibashi, H., Marubashi, K.: 2004, Structure of interplanetary magnetic cloud on April 16, 1999 and its origin estimated by fitting the torus-shaped flux rope model. Geophys. Res. Lett. 31, L21807. doi:10.1029/ 2004GL020702.

Lundquist, S.: 1950, Magnetohydrostatic fields. Ark. Fys. Ark. Phys. 2, $361-365$.

Miller, G., Turner, L.: 1981, Force free equilibria in toroidal geometry. Phys. Fluids 24, 363-365.

Romashets, E., Poedts, S.: 2007, Plasma flows around magnetic obstacles in the solar wind. Astron. Astrophys. 475, $1093-1100$.

Romashets, E.P., Vandas, M.: 2003, Interplanetary magnetic clouds of toroidal shapes. In: Wilson, A. (ed.): Proc. ISCS 2003 Symposium, Solar Variability as an Input to the Earth's Environment SP-535, ESA, Noordwijk, $535-540$.

Romashets, E.P., Vandas, M.: 2009, Linear force-free field of a toroidal symmetry. Astron. Astrophys. 499, $17-20$.

Sakai, J.-I., de Jager, C.: 1997, 3-D MHD simulation of X-type coalescence of two current-loops. Solar Phys. 173, $347-358$.

Verwichte, E., Nakariakov, V.M., Cooper, F.C.: 2005, Transverse waves in a post-flare supra-arcade. Astron. Astrophys. 430, L65-L68. 